\title{
Small bowel infarction mimicking Crohn's ileitis
}

\author{
P.J. Thuluvath, M.D. Feher and J. Wiggins \\ Westminster Hospital, Horseferry Road, London SW1, UK
}

\begin{abstract}
Summary: A case of superior mesenteric arterial embolic occlusion with radiological features mimicking small bowel Crohn's disease is reported.
\end{abstract}

\section{Introduction}

Despite the advances in vascular surgery and intensive care management, the mortality rate from acute intestinal ischaemia has not improved over the years. ${ }^{1,2}$ The advanced age of the patients, the toxic nature of bowel contents and co-existent cardiovascular disease may explain the high mortality rate; the delay in making the diagnosis also may be a major factor. Since there are no reliable laboratory or non-invasive radiological investigations, the diagnosis depends on a high index of clinical suspicion. We report a case illustrating the difficulty in making such a diagnosis, in particular, the problem of distinguishing between small bowel ischaemia and Crohn's disease.

\section{Case report}

A 73 year old woman with a previous history of depression and alcohol abuse was admitted with a history of watery diarrhoea and abdominal pain of 4 days duration. There were no previous gastrointestinal problems. On admission she was afebrile, well hydrated, normotensive with controlled atrial fibrillation and there were no cardiac murmurs. She had slight generalized abdominal tenderness without guarding; bowel sounds and rectal examination were normal. The following investigations were normal or negative; full blood count, urea and electrolytes, chest and abdominal radiographs, stool microscopy and culture, Clostridium difficile culture and toxin assay. The serum albumin was low at $25 \mathrm{~g} / \mathrm{l}$.

Intravenous fluids were started. On the third hospital day she developed a temperature of $38.2^{\circ} \mathrm{C}$, nausea, severe abdominal pain (without guarding), and passed loose stool with copious

Correspondence: P.J. Thuluvath, M.D., M.R.C.P., Department of Gastroenterology, West Middlesex University Hospital, Isleworth, Middlesex TW7 6F, UK. Accepted: 21 March 1991 blood; bowel sounds were normal. Blood cultures were negative, sigmoidoscopy, and subsequent histological examination of a rectal biopsy, were normal. Barium enema showed extensive diverticula with normal mucosal appearances. Despite continued abdominal pain and distension, she discharged herself against medical advice on the 10th hospital day.

She was readmitted the next day with further abdominal pain and diarrhoea; her general condition was unchanged. Plain abdominal radiograph and abdominal ultrasonography were normal. Haemoglobin $11 \mathrm{~g} / 1$, white cell count 8,900 and the erythrocyte sedimentation rate was $76 \mathrm{~mm} / \mathrm{h}$. Barium follow-through showed a long rigid segment of ileum with fissure ulcers consistent with Crohn's disease (Figure 1). Her symptoms continued and prednisolone, $40 \mathrm{mg}$ daily, was commenced with initial improvement. On the 30th hospital day she developed more severe abdominal pain with abdominal rigidity and absent bowel sounds; the abdominal radiograph showed two gas filled small bowel loops and fluid levels. At laparotomy a gangrenous ileum, caecum and part of the jejunum were excised. Histological examination of the proximal $30 \mathrm{~cm}$ of the resected small intestine showed ulceration lined by granulation tissue. The distal $20 \mathrm{~cm}$ of the small intestine and caecum showed infarction with a serosal fibropurulent exudate. Within the mesenteric and submucosal arteries fibrin thrombi were seen, some with evidence of recanalization. The appearances were those of systemic embolization with no evidence of Crohn's disease.

The patient died 7 days later despite supportive therapy. Post-mortem examination was not performed.

\section{Discussion}

The diagnosis of superior mesenteric artery occlusion is usually made clinically. ${ }^{3}$ This case illus- 


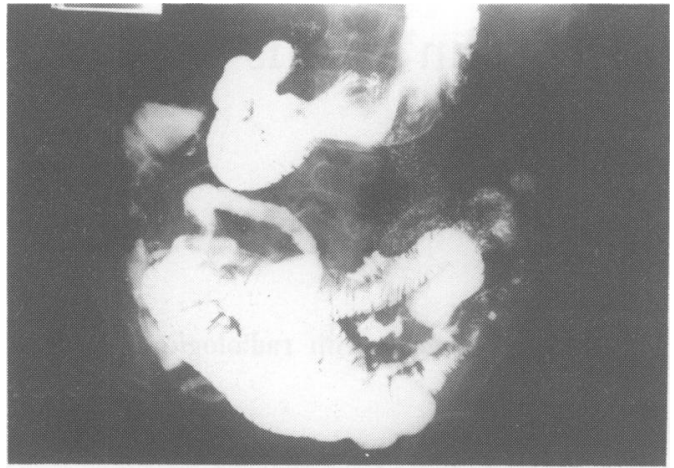

Figure 1 Barium follow-through showing a long rigid segment of ileum with fissure ulcers.

trates the difficulty in making the diagnosis, especially when the presentation is atypical.

Mesenteric arterial embolism commonly presents with abrupt onset of severe abdominal pain which is usually periumbilical but sometimes in the right upper quadrant. Vomiting and diarrhoea often follow, but bloody diarrhoea is a late feature which suggests that infarction has occurred. Initial clinical and radiological signs and laboratory tests are non-specific. Pain is usually disproportionate to the objective signs. Progression of infarction usually occurs in 12-24 hours, accompanied by signs of ileus and peritonitis.

In contrast, Crohn's disease usually presents with chronic and often persistent diarrhoea, lower abdominal pain and weight loss, although an acute presentation with either bloody diarrhoea, bowel obstruction or an abdominal mass is not uncommon in clinical practice. ${ }^{4,5}$ While bloody diarrhoea occurs in half the patients with Crohn's disease it is less common when only the small bowel is affected.

In our patient the subacute onset, the absence of tachycardia and hypotension, and the radiological changes highly suggestive of Crohn's disease were all misleading. The progression of the infarction over a period of 30 days was also unusual. Multiple embolization of distal vessels may explain the sub-acute onset and slow progression in this patient.

Radiological appearances of incomplete small bowel infarction mimicking those of Crohn's disease are not documented in the literature, perhaps because patients with small bowel infarction usually do not have barium studies. However, in large bowel ischaemia radiological appearances indistinguishable from ulcerative colitis and Crohn's colitis have been reported. In a series of 8 patients with ischaemic colitis, Eisenberg et al. observed a variety of radiological findings includ- ing thumbprinting, shallow and deep ulceration, pseudopolyps, toxic megacolon and strictures on plain abdominal radiographs or barium enema; but none of the patients had terminal ileal involvement. ${ }^{6}$ Thumbprinting, which is usually associated with ischaemic colitis, may also be seen in Crohn's colitis. $^{7,8}$

The small bowel histology in the case reported had no features of Crohn's disease. However, it may be difficult to differentiate histologically between inflammatory bowel disease and low grade ischaemic colitis, as the presence of crypt abscesses and epithelioid granulomas does not exclude ischaemic colitis. In an elderly subject the subsequent clinical progression is considered a better guide than the evolution of histology. ${ }^{3}$

The age of presentation of ischaemic bowel differs greatly from Crohn's disease. The mean age of presentation of Crohn's disease is around 32 years (range 5-83) with a second peak around 60-65 years; by contrast, ischaemic bowel is usually seen in subjects over 65 years. ${ }^{1,9}$ While it is possible that few patients with ischaemic bowel are included in the Crohn's disease group it may not entirely explain the second peak. It is of interest to note the recent concept linking Crohn's disease to multifocal infarction mediated by mesenteric vasculitis. $^{10}$

Arterial embolism and thrombosis are the twळ major causes of acute mesenteric arterial occlusion The less common causes are non-occlusive ischaes mia, venous thrombosis, dissecting aortic aneurysm and fibromuscular hyperplasia. The risk factors include heart failure, digitalis overdose, immunosuppression, major sepsis and contraceptive pills. Atherosclerosis is considered to be a common cause for acute mesenteric arterial thrombosis in elderly subjects. Causes of emboli include atherosclerotic plaques, endocarditis, valvular prosthesis or, rarely, left atrial myxomas. Embolic occlusions account for $25-30 \%$ of acute intestinal ischaemia; about $90 \%$ of emboli arise from heart often associated with atrial fibrillation, and was the most likely source in our patient.,11

The mortality rate from embolic occlusion of the superior mesenteric artery is $60-90 \%$ and is due to the patient's advanced age, the delay in diagnosis and co-existent cardiovascular problems. ${ }^{1,2}$ In our present case it is possible that an earlier diagnosis, before complete bowel infarction had occurred, could have prevented the death. The management of acute intestinal ischaemia has been reviewed recently by Marston. ${ }^{2}$

This case report illustrates the difficulty in correctly diagnosing ischaemia and infarction of bowel in elderly patients with sudden onset of diarrhoea and abdominal pain. The presence of radiological findings which were characteristic of Crohn's disease in this case was misleading. 


\section{Acknowledgements}

We wish to thank Professor A.F. Lant and Dr J. Gleeson for their permission to report this case.

\section{References}

1. Wilson, C., Gilmour, D.G. \& Imrie, C.W. Acute superior mesenteric ischaemia. Br J Surg 1987, 74: 279-281.

2. Marston, A. Acute intestinal ischaemia. Br Med J 1990, 301: 1174-1176.

3. Moosa, A.R., Shackford, S. \& Sise, M.J. Acute intestinal ischaemia. In: Marston, A. (ed.) Vascular Disease of the Gut. Arnold, London, 1986, pp. 64-85.

4. Farmer, R.G., Hawk, W.A. \& Turnbull, R.B.Jr. Clinical patterns in Crohn's disease. A statistical study of 615 cases. Gastroenterology 1975, 68: 627-635.

5. Mekhajian, H.S., Switz, D.M., Watts, H.D. et al. Clinical features and natural history of Crohn's disease. Gastroenterology 1979, 77: 898-906.

6. Eisenberg, R.L., Montogomery, C.K. \& Marguilis, A.R. Colitis in the elderly: ischaemic colitis mimicking ulcerative and granulomatous colitis. AJR 1979, 133: 1113-1118.
7. Lichtenstein, J.E. The radiologic-pathologic correlation of inflammatory bowel disease. Radiol Clin North Am 1987, 25: 3-24.

8. Marshak, R.H. \& Lindner, A.E. Radiologic diagnosis of chronic ulcerative colitis and Crohn's disease of the colon. In : Kirsner, J.B. \& Shorter, R.G. (eds) Inflammatory Bowel Disease. Lea \& Febiger, Philadelphia, 1975, p. 246.

9. Mendeloff, A.I. \& Calkins, B.M. The epidemiology of idiopathic inflammatory bowel disease. In: Kirsner, J.B. \& Shorter, R.G. (eds) Inflammatory Bowel Disease. Lea \& Febiger, Philadelphia, 1988, pp. 3-34.

10. Wakefield, A.J., Sawyer, A.M., Dhillon, A.P. et al. Pathogenesis of Crohn's disease: multifocal gastrointestinal infarction. Lancet 1989, ii: 1057-1062.

11. Kairalvoma, M.I., Karkola, P., Heikinnen, E. et al. Mesenteric infarction. Am J Surg 1977, 133: 188-193. 